\title{
Image Classification for Silkworm using Deep Neural Network-Keras
}

doi : https://doi.org/10.32628/CSEIT2173139

\section{Nishali M Suvarna, Sudarshan K, Nisha S Ail}

Department of Computer Science, Srinivas Institute of Technology, Valachil, Mangaluru, Karnataka, India

\begin{abstract}
Article Info

Volume 7, Issue 3

Page Number: 658-663

Publication Issue :

May-June-2021

Article History

Accepted : 12 June 2021

Published : 20 June 2021

Sericulture is a practice of cultivating silkworms to produce silk. This project is used to classify silkworms into diseased and undiseased, to increase the production of the silkworms which results in increasing the production of rich silk. To identify the unhealthy silkworm in the larvae stage so as to stop the destruction of entire batch of the silkworm. The high accuracy model is built .To extract the general features and then classify them under multiple based upon the features detected. To increase the quality and quantity of production of the silkworms. This project will help the farmers financially as the production increases.

Keywords: Image Classification, Convolutional Neural Network, Keras, Deep Learning.
\end{abstract}

\section{INTRODUCTION}

Sericulture is an important agro-industry, playing an important role in the rural and urban economy of several countries. Effective utilization of overall sericulture practice requires additional eco-friendly approaches such as application of enzymes for the better product yields. This project is to classify or identify the silk worm using CNN that optimizes seed production of silkworms, which increases silk production by building a desired high accuracy model for silkworm classification and also to identify the healthy and unhealthy silkworm to improve the production of silk.

There are wide variety of diseases that may be seen during the production of the silkworm. These diseases if occurs in one silkworm it will spread throughout entire batch and destroy all the silkworms. In order to avoid this the infected silkworm has to be detected in initial stage and should be separated from the rest. The Diseases of silkworm include 1. Grasserie is a viral disease where Inter segmental swelling appears in the silkworm and the colour of the body becomes yellowish. Infected larval body ruptures easily and turbid while haemolymph oozes out. 2. Flacherie is a disease that is common during Summer and Rainy seasons. Silkworm gets infected by eating contaminated mulberry leaf. The infection can also take place through injuries/cuts/wounds. 3. Muscardine is a fungal disease that commonly occurs during rainy and winter seasons. During this, larva vomits and turns flaccid. 4. Pebrine is caused either through eggs or by eating contaminated mulberry leaf. Black pepper like spots appear on the body of the infected worms. White postules appear on the silkgland. 
Silk is mainly used in garments and household items, but it is also employed in unexpected ways, such as bicycle tires and in medicine. Silk is a staple of many gowns and dresses. The material's strength and nuances with color make it ideal for accessories. It is great material for scarves for both decoration and for warmth. Silk was originally used for parachutes for its strength and elastic properties. The silk does not cause an autoimmune response and cannot be absorbed by the human body therefore it is useful in medicine. Most of the farmers earn by practicing sericulture. The profit rate is high as the silk are most valuable fibre. Silk is called as Queen of Textiles. The high quality silkworm is in high demand in the market. Women are also indulged in the production. There is no age to cultivate silkworm. Anyone in the family can do this and earn through rearing the silkworm.

In the present situation, high quality silkworm and defective silkworm are identified manually. This identification process is inefficient and time consuming. In recent years, convolutional neural network (CNN) has achieved high success and gradually become the main method in the area of image classification.

In this project we are going to build a convolution neural network to solve the problem of identifying diseased and undiseased silkworms. Keras would used for model building and all the code would be implemented on google colab.

\section{$\mathrm{CNN}$ (convolutional neural network)}

The name "convolutional neural network" indicates that the network employs a mathematical (convolution) operation. Convolution is a specialized kind of linear operation. Convolutional networks are simply neural networks that use convolution in place of general matrix multiplication in at least one of their layers. A convolutional neural network consists of an input and an output layer, as well as multiple hidden layers. The hidden layers of a CNN typically consist of a series of convolutional layers that convolve with a multiplication or other dot product. The activation function is commonly a RELU layer, and is subsequently followed by additional convolutions such as pooling layers, fully connected layers and normalization layers, referred to as hidden layers because their inputs and outputs are masked by the activation function and final convolution. The final convolution, in turn, often involves backpropagation in order to more accurately weight the end product.

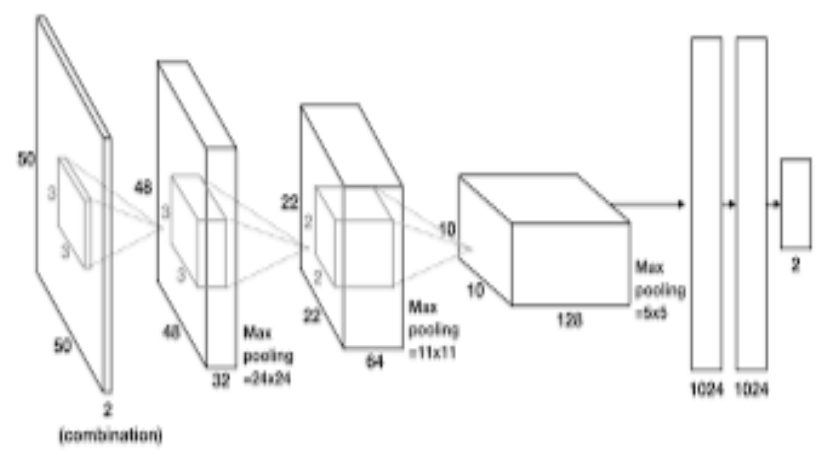

Fig 1. Basic CNN architecture

\section{LITERATURE RIVIEW}

Many problems in computer vision were saturating on their accuracy before a decade. However, with the rise of deep learning techniques, the accuracy of these problems drastically improved. One of the major problem was that of image classification, which is defined as predicting the class of the image. Cat and Dog image classification is one such example of where the images of cat and dog are classified. This paper aims to incorporate state-of-art technique for object detection with the goal of achieving high accuracy. A convolutional neural network is been build for the image classification task.In this project this techniques used to classify the diseased and 
undiseased silkwarms [1]. The silkworm is the larva or caterpillar of the domesticated silk moth, Bombyx mori. It is an important economic insect since it is a producer of silk. Flacherie is a syndrome associated with bacterial diseases. In the present study diseased silkworms were collected, dissected to separate the contents of foregut, midgut, and hindgut. The samples were serially diluted and from which 10-5, 10-6 dilutions were plated onto nutrient agar plates. The predominant colonies were selected and identification procedures such as colony morphology, staining property and biochemical tests. As a result the organisms identified were Bacillus subtilis, Streptococcus pneumoniae, Staphylococcus aureus, E.coli, Pseudomonas fluorescence, Bacillus cereus and Klebsiella cloacae. Hence the study concludes with this bacterial identification which is responsible for bacterial flacherie in silkworms. So if a measure is taken to control these bacterial species the disease incidence in silkworms can be reduced which in turn increase the economics [2]. With the development of artificial intelligence, deep learning is more and more widely used in image recognition. As a representative deep learning algorithm, convolutional neural network has been widely used due to its excellent performance in image processing. It is widely used in various fields and has made great achievements. Image recognition is the most important problem in the development of computer vision. Since computers can recognize objects, computer vision can be used in many fields[3]. Biologically inspired ideas are important in image processing. Not only does more than $80 \%$ of the information received by humans comes from the visual system, but the human visual system also gives its fast, accurate, and efficient image processing capability. In the current image classification task, convolutional neural networks (CNNs) focus on processing pixels and often ignore the semantic relationships and human brain mechanisms. With the development of image analysis and processing techniques, the information in the image is becoming increasingly complicated. Humans can learn about the characteristics of objects and the relationships that occur between them to classify the images. It is a significant characteristic that sets humans apart from the modern learning-based computer vision algorithms [4].Deep Learning models have made incredible progress in discriminative tasks. This has been fueled by the advancement of deep network architectures, powerful computation, and access to big data. Deep neural networks have been successfully applied to Computer Vision tasks such as image classification, object detection, and image segmentation thanks to the development of convolutional neural networks (CNNs). These neural networks utilize parameterized, sparsely connected kernels which preserve the spatial characteristics of images[5]. Due to object detection's close relationship with video analysis and image understanding, it has attracted much research attention in recent years. Traditional object detection methods are built on handcrafted features and shallow trainable architectures. Their performance easily stagnates by constructing complex ensembles which combine multiple low-level image features with high-level context from object detectors and scene classifiers. With the rapid development in deep learning, more powerful tools, which are able to learn semantic, high-level, deeper features, are introduced to address the problems existing in traditional architectures. These models behave differently in network architecture, training strategy and optimization function, etc [6]. Recently, image classification is growing and becoming a trend

among technology developers especially with the growth of data in different parts of industry such as e-commerce,

automotive, healthcare, and gaming. The most obvious

example of this technology is applied to Facebook. Facebook now can detect up to $98 \%$ accuracy in order to identify your face with only a few tagged 
images and classified it into your Facebook's album. The technology itself almost beats the ability of human in image classification or recognition (What is the Working of Image Recognition and How it is Used, 2017) [7].The domesticated silkworm, Bombyx mori, is an important economic insect for silk production. There are several types of silkworm diseases, and they cause great economic losses to the sericulture industry. Among them, B. mori nucleopolyhedrovirus (BmNPV) disease is the most serious (Jiang and Xia, 2014; Xu et al., 2015). BmNPV disease is acute, and the duration from infection to illness is only approximately $96 \mathrm{~h}$. The body wall of infected silkworms breaks easily and, thus, the pathogen pollutes the mulberry leaves on the silkworm bed, which leads to the rapid spread of the disease. So far, BmNPV has not been controlled effectively by therapeutic agents (Bao et al., 2009). [8].

\section{PROBLEM STATEMENT}

At present, the diseased and undiseased silkworm are identified manually. This is very inefficient method. If one is not able to predict the silkworms correctly and classify them, the entire batch of silkworm will be in danger. This will lead to loss for the farmers and also, the production of next batch of silkworm will be difficult as it is dependant on the previous batch. This chaining system will be disturbed and it will loose the quality of the silkworm along with its quantity.

\section{METHODOLOGY}

\section{A. Proposed Model}

Classifier is the name given to the Sequential model. The model's first layer is a Conv2D layer. Since, it is the first layer of the model, input shape of the images that are going to be supplied to the model is being mentioned. Next layer is a batch normalization layer. Then one activation layer corresponding to the conv2d layer. Further there is another set of conv2d, batch normalization and activation layer with different number of kernels in the conv2d layer. After that a max Pooling layer is there and then a dropout layer is there.

The same set of layers is again repeated with different number of kernel's and dropout rate. The convolution layers end with this set. Next are the fully connected layer.

The Sequential model API is used to build model. The sequential API allows you to create models layer-by-layer. The 'add()' function to add layers to our model. The model needs to know what input shape it should expect. For this reason, only the first layer in a Sequential model needs to receive information about its input shape.

Dropout layer consists in randomly setting a fraction rate of input units to 0 at each update during training time, which helps prevent overfitting.

Activation layer are used to apply the activation function to the output of that layer. The purpose of the activation function is to introduce non-linearity into the output of a neuron. Relu and sigmoid activation are used in the model.

Batch Normalization is used for improving the speed, performance, and stability of artificial neural networks. Batch normalization is a method we can use to normalize the inputs of each layer and achieve faster convergence.

Maximum pooling, or max pooling, is a pooling operation that calculates the maximum, or largest, value in each patch of each feature map. The results are down sampled or pooled feature maps that highlight the most present feature in the patch.

In Conv2D layer, a kernel, convolution matrix, or mask is a small matrix. It is used for blurring, sharpening, embossing, edge detection, and more. This is accomplished by doing a convolution between a kernel and an image. This layer creates a convolution kernel that is convolved with the layer input to produce a tensor of outputs. 
Then comes the fully connected layers. It contains only 2 layers. First one is the global average pooling layer to minimize overfitting by reducing the total number of parameters in the model. Second layer and the final layer is the Dense layer with sigmoid activation.

Global Average Pooling 2D layer is used to minimize overfitting by reducing the total number of parameters in the model. GAP layers perform a more extreme type of dimensionality reduction, where a tensor with dimensions $\mathrm{h} \times \mathrm{w} \times \mathrm{d}$ is reduced in size to have dimensions $1 \times 1 \times d$. GAP layers reduce each $\mathrm{h} \times \mathrm{w}$ feature map to a single number by simply taking the average of all hw values.

During the model compilation, the optimizer algorithm, loss function and the list of metrics are parameters which are to be taken care of. The model trained for 11 epochs. Below is the table showing the end result of training i.e train accuracy, test accuracy, train loss, test loss and epochs.

TABLE I. Training Result Per Epoach

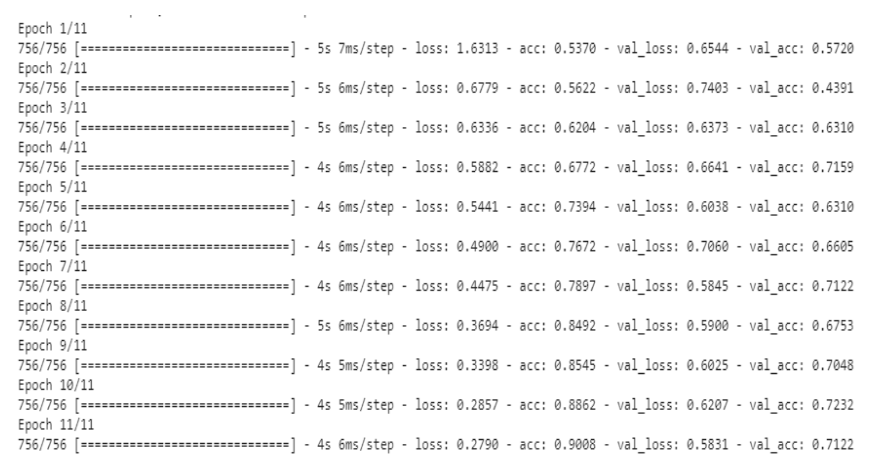

\section{B. Dataset}

To perform deep learning method for our proposed work, we use silkworm image dataset. Where the dataset consists of 1000 silkworm images in with 800 images for training and 200 silkworm images for Testing, Our Dataset contains two categories: Diseased and Undiseased as shown in Fig. 2. There are at least 500 images in each category. For the assessment, we divided the images into $8: 2$ train/test sets for each category. Since the images were chosen at random for each collection, the accuracy varies depending on which image was chosen, and we report the average accuracy and loss.

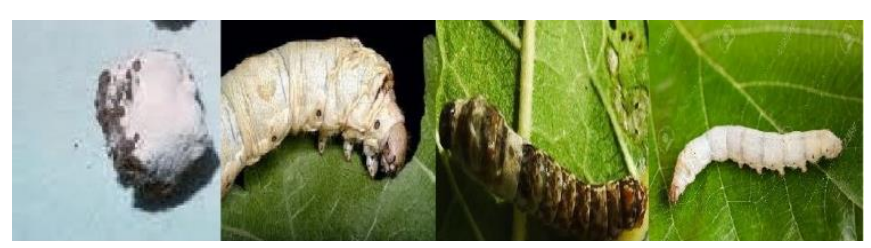

Fig. 2. Example Images from dataset of train and test (a)test-diseased (b)test-undiseased (c) train-diseased (d) train-undiseased

\section{RESULT}

The image classification of silkworm as diseased and undiseased is successfully obtained. By using just small amount of dataset $75 \%$ accuracy has been obtained. If more images are used then accuracy will also increase. It is seen that image classification using $\mathrm{CNN}-$ Keras is very efficient and easy to implement.

\section{CONCLUSION}

In this paper we build deep convolutional neural network for image classification (silkworm images). Despite of using only the subset of the images an accuracy of $75 \%$ was obtained. If the whole dataset was being used the accuracy would have been even better. There is no such technology currently in existence, which can be used to classify the diseased and undiseased silkworm. Such technology will help the problems faced by the farmers while cultivating silkworms, especially when the disease spread to the entire batch and destroy them and thus, in maintaining the quality and quantity of the silkworm. 


\section{FUTURE SCOPE}

In this project the input is images. Further we can use sensors and some networks and convert this into live project. The farmers can directly use the sensors and see whether the silkworm is diseased or undiseased within few seconds instead of taking pictures and loading it into the system.

\section{REFERENCES}

[1]. Tushar Jajodia, Pankaj Garg ,"Image Classification - Cat and Dog Images".

[2]. S. Sakthivel , C. Angaleswari , P. U. Mahalingam,Department of Biology, Gandhigram Rural Institute- Deemed University, Gandhigram, Dindigul ," Isolation and identification of bacteria responsible for flacherie in silkworms".

[3]. Lan Chen, Zhiyuan Jiang, and Zhiyong Wang, "Image Recognition Based on Convolution Neural Network with Small Data Setl".

[4]. Dehai zhang1, Menglong cui1, Yun yang 1 , Po yang 1,2, (member, ieee), Cheng xie 1 , (member, ieee), Di liu 1 , Beibei yu1, and Zhibo chen1 "Knowledge Graph-Based Image Classification Refinement".

[5]. Taghi M.Khoshgoftaar,Connor Shorten ,"A survey on Image Data Augmentation for Deep Learning".

[6]. Zhong-Qiu Zhao, Member, IEEE, Peng Zheng, Shou-tao $\mathrm{Xu}$, and Xindong $\mathrm{Wu}$, "Object Detection with Deep Learning: A Review".

[7]. Mohd Azlan Abu, Nurul Hazirah Indra , Abdul Halim Abd Rahman , Nor Amalia Sapiee and Izanoordina Ahmad1, "A study on Image Classification based on Deep Learning and Tensorflow".

[8]. Yang Qiong, Xing Dong XuLi , Quing Rong, Xiao Yang, Ye Ming Qing, "Standard method for detecting Bombyx mori nucleopolyhedrovirus disease-resistant silkworm varieties".

\section{Cite this article as :}

Nishali M Suvarna, Sudarshan K, Nisha S Ail , "Image Classification for Silkworm using Deep Neural Network-Keras", International Journal of Scientific Research in Computer Science, Engineering and Information Technology (IJSRCSEIT), ISSN : 24563307, Volume 7, Issue 3, pp.658-663, May-June-2021. Available at

doi $\quad$ : https://doi.org/10.32628/CSEIT2173139 Journal URL : https://ijsrcseit.com/CSEIT2173139 